Supplement of Geosci. Model Dev., 14, 735-761, 2021

https://doi.org/10.5194/gmd-14-735-2021-supplement

(C) Author(s) 2021. This work is distributed under

the Creative Commons Attribution 4.0 License.

(c) (1)

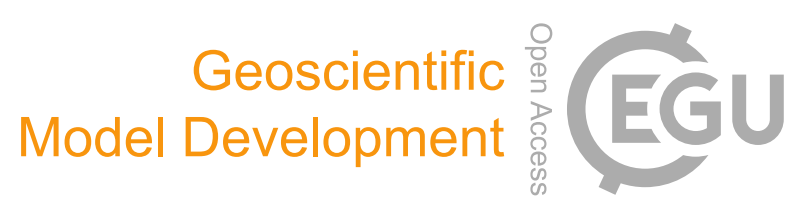

Supplement of

\title{
CoupModel (v6.0): an ecosystem model for coupled phosphorus, nitrogen, and carbon dynamics - evaluated against empirical data from a climatic and fertility gradient in Sweden
}

Hongxing He et al.

Correspondence to: Hongxing He (hongxing-he@ hotmail.com) and Annemieke I. Gärdenäs (annemieke.gardenas@ bioenv.gu.se)

The copyright of individual parts of the supplement might differ from the CC BY 4.0 License. 


\section{Global sensitivity analysis of all new $P$ parameters and relevant $\mathbf{N}$ parameters}

\section{Parameters and the associated ranges}

The $\mathrm{P}$ parameters introduced in Coup-CNP, along with $\mathrm{N}$ process parameters relevant for plant photosynthesis, nutrient uptake parameters and fungal growth parameters, were selected for a global sensitivity analysis (Table S.1). Overall, 34 parameters were analyzed, with these parameters further grouped into 13 categories: (1) Initial value $\left(\operatorname{Ini}_{P, h}\right) ;(2) \mathrm{N}$ uptake $\left(o_{\text {uptNhumus }}\right)$; (3) P uptake (ouptPhumus, $p_{\text {iavail }}$ ); (4) Source $\left(k_{w}, n_{H}, p H_{\text {opt }}\right)$; (5) Partitioning ( $p_{\text {max ads }}, c_{50, a d s}$ ); (6) Photosynthesis ( $\left.p_{c p, o p t}, n_{c n, o p t}, p_{c p, t h}, n_{c n, t h}\right)$; (7) GPP allocation ( $\left.p_{\text {avail }}, n_{\text {avail }}, p_{\text {fopt }}\right)$; (8) Fungi $\left(k_{r m}, p_{\text {lrate }}\right)$; (9) $\mathrm{P}$ demand to fungi $\left(p_{i \text {,rate }}, p_{\text {cpfungimax }}, p_{\text {cpfungimin }}, p_{\text {olit rate }}, p_{\text {ohum, rate }}\right) ;(10) \mathrm{N}$ demand to fungi $\left(n_{N H 4 r a t e}\right.$, $\left.n_{N O 3 r a t e}, n_{\text {olit, rate }}, n_{\text {ohum, rate }}\right)$; (11) P transformation $\left(c p_{m}, m_{\text {retain }}\right)$; (12) P demand to plant ( $c p_{\text {leaf, min }}$, $\left.c p_{\text {stem, } \min }, c p_{\text {croot, } \min }, c p_{\text {root, } \min }\right)$; and (13) Erosion $\left(p_{\text {base }}, p_{\Delta}, q_{t h r}\right)$. The parameter ranges were obtained by varying the default values reported in Table 3 by $\pm 50 \%$ (Table S.1). The default values were used as a reference since they have been calibrated against observations of forest growth, leaf nutrient ratios and nutrient leaching.

The initial soil $\mathrm{C}$ and soil $\mathrm{N}$ values (Table 1) for each region were kept constant. Due to uncertainties in the soil $\mathrm{P}$ data for deeper soil layers, we included the initial value of soil humus $\mathrm{P}$ in the global sensitivity analysis. The initial value of total organic $\mathrm{P}$ in humus varied from 8.45 to $21.16 \mathrm{~g} \mathrm{P} \mathrm{m}^{-2}$ for the $64^{\circ} \mathrm{N}$ region (Table S.1). This defined total $\mathrm{P}$ range translates to a total soil C/P ratio of $315-788$, which is similar to the synthesized literature value (N/P between 10 to 25 , see section 3.2) for this region.

For the $56^{\circ} \mathrm{N}$ region, the initial value of soil humus $\mathrm{P}$ varied from 18.42 to $53.9 \mathrm{~g} \mathrm{P} \mathrm{m}^{-2}$ (Table S.1), translating to a soil C/P ratio of 188-550. This range was set to be wide enough to cover the current synthesized literature value 198-495 for the region, along with additional data from the Swedish Forestry Agency concerning the humus layer (e.g. C/P of 188 in Gynge, Table 1). To summarize, the ranges of initial soil $\mathrm{P}$ tested in the sensitivity analysis cover the C/P ranges of the regional sites presented in data from the Swedish Forestry Agency and relevant literature.

\section{Model design}

We conducted a Monte Carlo-based global sensitivity analysis for 34 parameters (Table S.1) for two regions: the northernmost $64^{\circ} \mathrm{N}$ region, characterized by $\mathrm{N}$ limitation, and the southernmost $56^{\circ} \mathrm{N}$ region, characterized by $\mathrm{P}$ limitation. The probability distribution function for each parameter was assumed to be uniform. Random sampling was used to generate random sets of parameter values. Overall, 600 runs were conducted for each region. We evaluated the responses of a number of model variables to changes in the analyzed parameters. The importance of the 34 parameters was analyzed based on changes in the $\mathrm{C}, \mathrm{N}$, and $\mathrm{P}$ components that are vital for ecosystem functioning. More specifically, we assessed the magnitude of changes in ecosystem $\mathrm{C}$ change, total $\mathrm{C}$ harvested, plant $\mathrm{C}$ change, soil $\mathrm{C}$ change, plant $\mathrm{N}$ change, soil $\mathrm{N}$ change, plant $\mathrm{P}$ change, soil $\mathrm{P}$ change, and N/P response on GPP. Correlation analysis (Pearson correlation coefficient, r) was used to rank the parameters based on importance. The analysis proceeded as follows: first, model performance indicators including correlation of determination, $\mathrm{R}^{2}$, and mean error with respect to the reference model run (i.e. Fig. 2) were estimated for each model run; then, Pearson correlation coefficient ( $r$ ) was calculated from the parameter and the model performance indicators. Thus, a high $r$ value 
between a parameter and the $\mathrm{R}^{2}$ (or ME) value of a selected variable means that the parameter is important in regulating the dynamics (or magnitude) selected variable. We chose $r \geq 0.2$ or $\leq$ -0.2 as a threshold for parameter importance.

\section{Parameter sensitivity}

The global sensitivity analysis results identified three important parameters for the regulation of forest ecosystem $\mathrm{C}, \mathrm{N}$ and $\mathrm{P}$ cycling. These include initial soil organic $\mathrm{P}\left(\operatorname{Ini_{P,h}}\right)$, the coefficient for short-cut $\mathrm{N}$ uptake from the humus pool $\left(o_{\text {uptNhumus }}\right)$ and the coefficient for shortcut P uptake from the humus pool ( $\left.o_{\text {uptPhumus }}\right)$ (Table S.2, Table S.3). The northern regions were more sensitive to changes in $o_{\text {uptNhumus, }}$ a finding which reflects the N-limited nature of the $64^{0} \mathrm{~N}$ region, whereas the southern $56^{0} \mathrm{~N}$ region was more sensitive to $I n i_{P, h}$ due to $\mathrm{P}$ limitation (Figs. S.1, S.2, S.3, S.4).

The first parameter with a large impact on model output was the initial vale of soil $\mathrm{P}$, which determines the $\mathrm{P}$ content in soil organic matter. The importance of the initial value of humus $\mathrm{P}, I_{i i_{,},}$, again confirms that the $\mathrm{C} / \mathrm{P}$ and $\mathrm{N} / \mathrm{P}$ ratios in organic matter have crucial roles in determining the ecosystem $\mathrm{C}, \mathrm{N}$ and $\mathrm{P}$ fluxes and pool sizes, as we concluded in the main paper. In addition, very few parameter from the source category (parameters controls weathering rates, e.g. $p H_{\text {opt }}$ ) showed a noticeable impact on model output (Table S.2). This confirms that the internal cycling of $\mathrm{P}$ is more important in regulating ecosystem $\mathrm{C}, \mathrm{N}$ and $\mathrm{P}$ dynamics than the current weathering inputs in Swedish forests.

Parameters related to N/P uptake (ouptNhumus, $o_{\text {uptPhumus}}$ ) and $\mathrm{P}$ transformation $\left(c p_{m}\right)$ also significantly affected model output. These parameters represent processes that directly control $\mathrm{N}$ and $\mathrm{P}$ availability or release from mineralization, which are important to regulating photosynthesis and forest growth (Table S.2, Table S.3).

The third set of parameters identified through the sensitivity analysis covered plant growth and dynamics, including three categories: $\mathrm{P}$ demand to $\mathrm{P}\left(c p_{\text {leaf } \text { min }}, c p_{\text {leaf, root }}\right)$; Photosynthesis $\left(n_{c n, o p t}\right.$, $\left.n_{c n, t h}, p_{c p, o p t}, p_{c p, o p t}\right)$; and GPP allocation ( $\left.p_{\text {fopt }}\right)$ (Table S.2, Table S.3). These parameters regulate $\mathrm{C}$ allocation within the plants, plant stoichiometry, as well as plant-fungi symbiosis. Interestingly, total harvested $\mathrm{C}$ was negatively correlated with the $\mathrm{C}$ allocated to symbiotic fungi $\left(p_{\text {fopt }}\right)$ for both regions.

Changes in many parameters were found to impact more than one simulated variable. The sign of the correlation between a parameter and soil nutrient (N/P) change, is flipped for that of plant nutrient (N/P) change (Table S.2, Table S.3). This is expected as the nutrients are generally relocate between plant and soil, with minor inputs. The interaction between $\mathrm{N}$ and $\mathrm{P}$ was identified in the sensitivity analysis. For instance, changes in two parameters $\left(\operatorname{Ini}_{P, h}, c p_{m}\right)$ regulating $\mathrm{P}$ availability in the soil consistently had a large influence on the simulated response of N on GPP (Table S.2, Table S.3).

The global sensitivity analysis highlighted the importance of initial organic $\mathrm{P}$ and the short-cut uptake coefficients. We conducted an additional analysis by removing the three key parameters $\left(I n i_{P, h}, o_{\text {uptNhumus, }}\right.$ OuptPhumus $)$ from the global sensitivity analysis. We then re-ran the model, 300 runs for the northernmost $64^{\circ} \mathrm{N}$ region, with the remaining 31 parameters. The results are shown in Table S.4. This analysis identified the parameters with the largest effect on model output to be those that had been included in the initial second and third set of sensitive 
parameters, notably, $\mathrm{P}$ transformation $\left(c p_{m}\right)$, plant $\mathrm{P}$ demand $\left(c p_{\text {leaf } \min }, c p_{\text {leaf, root }}\right)$, Photosynthesis $\left(n_{c n, o p t}, n_{c n, t h}, p_{c p, o p t}, p_{c p, o p t}\right)$ and GPP allocation $\left(p_{f o p t}\right)$ (Table S.2, Table S.3).

The fourth set of key parameters represent fungi-related processes. Among these, fungal respiration rate $\left(k_{r m}\right)$, GPP allocation to fungi $\left(p_{\text {fopt }}\right)$, fungal litter rate ( $\left.p_{\text {lrate }}\right)$, and $\mathrm{N}$ demand to fungi ( $n_{\text {NO3rate }}, n_{\text {NH4rate }}$ ) were shown to have the most significant effects on model output (Table S.4). Parameters in the partitioning $\left(c_{50, a d s}, p_{\text {max }}, a d s\right)$ and weathering $\left(p H_{o p t}, k_{w}\right)$ categories had the least significant effect on model output (Table S.4). 
Table S.1 List of parameters, and the corresponding ranges, used in Monte Carlo based sensitivity analysis. For a detailed explanation, including equations, for the parameters, see section 3 in the paper. Please note that the initial value for total humus $\mathrm{P}$ had different ranges in the $64^{0} \mathrm{~N}$ and $56^{\circ} \mathrm{N}$ region.

\begin{tabular}{|c|c|c|c|c|c|c|}
\hline Category & Symbol & Parameter & Equation & Min & Max & Unit \\
\hline Initial value & $\operatorname{Ini_{P,h}}$ & $\begin{array}{l}\text { Initial value for total } \\
\text { organic } \mathrm{P} \text { of humus }\end{array}$ & - & 8.45 & 53.9 & $\mathrm{~g} \mathrm{P} \mathrm{m}^{-2}$ \\
\hline$N$ uptake & $o_{\text {uptNhumus }}$ & $\begin{array}{l}\text { Coefficient for } \\
\text { shortcut } \mathrm{N} \text { uptake } \\
\text { from humus }\end{array}$ & - & $2.50 \times 10^{-6}$ & $1.50 \times 10^{-5}$ & day $^{-1}$ \\
\hline P uptake & $O_{\text {uptPhumus }}$ & $\begin{array}{l}\text { Coefficient for } \\
\text { shortcut } \mathrm{P} \text { uptake } \\
\text { from humus }\end{array}$ & - & $5.00 \times 10^{-6}$ & $2.75 \times 10^{-5}$ & day $^{-1}$ \\
\hline Source & $k_{w}$ & $\begin{array}{l}\text { Integrated } \\
\text { weathering rate }\end{array}$ & (1) & $4 \times 10^{-7}$ & $1.2 \times 10^{-6}$ & day $^{-1}$ \\
\hline Source & $n_{H}$ & $\begin{array}{l}\text { Weathering } \mathrm{pH} \\
\text { response coefficient }\end{array}$ & (4) & 0.135 & 0.405 & - \\
\hline Source & $\overline{p H_{\text {opt }}}$ & $\begin{array}{l}\text { Weathering } \mathrm{pH} \\
\text { response base } \\
\text { coefficient }\end{array}$ & (4) & 3.5 & 10.5 & - \\
\hline Partitioning & $p_{\text {max }, a d s}$ & $\begin{array}{l}\text { Langmuir max } \\
\text { sorption capacity }\end{array}$ & $(5)$ & 0.0001 & 0.0003 & $\begin{array}{l}\mathrm{gPg} \\
\text { soil }^{-1}\end{array}$ \\
\hline Partitioning & $c_{50, a d s}$ & $\begin{array}{l}\text { Langmuir half } \\
\text { saturation coefficient }\end{array}$ & (5) & $2.5 \times 10^{-5}$ & $7.5 \times 10^{-5}$ & $\mathrm{~g} \mathrm{P} \mathrm{m}^{-2}$ \\
\hline Photosynthesis & $p_{c p, o p t}$ & C/P optimal (leaf) & $(8)$ & 125 & 375 & $\mathrm{gC} \mathrm{gP}^{-1}$ \\
\hline Photosynthesis & $n_{\text {cn,opt }}$ & C/N optimal (leaf) & - & 12.5 & 37.5 & $\mathrm{gC} \mathrm{gN}^{-1}$ \\
\hline Photosynthesis & $p_{c p, t h}$ & C/P threshold (leaf) & $(8)$ & 400 & 800 & $\mathrm{gC} \mathrm{gP}^{-1}$ \\
\hline Photosynthesis & $n_{c n, t h}$ & $\mathrm{C} / \mathrm{N}$ threshold (leaf) & - & 37.5 & 75 & ${\mathrm{gC} \mathrm{gN}^{-1}}^{-}$ \\
\hline GPP allocation & $p_{\text {avail }}$ & $\begin{array}{l}\text { Coefficient for the } \\
\text { reduction of C } \\
\text { allocation to fungi } \\
\text { under high P } \\
\text { availability }\end{array}$ & $(9)$ & 0.00045 & 0.00135 & - \\
\hline GPP allocation & $n_{\text {avail }}$ & $\begin{array}{l}\text { Coefficient for the } \\
\text { reduction of } \mathrm{C} \\
\text { allocation to fungi } \\
\text { under high } \mathrm{N} \\
\text { availability }\end{array}$ & & 0.000195 & 0.000585 & - \\
\hline GPP allocation & $p_{\text {fopt }}$ & $\begin{array}{l}\text { The optimum ratio } \\
\text { for } \mathrm{C} \text { allocation } \\
\text { between fungi and } \\
\text { root }\end{array}$ & $(11)$ & 0.11 & 0.33 & - \\
\hline Fungi & $k_{r m}$ & $\begin{array}{l}\text { Fungal respiration } \\
\text { coefficient }\end{array}$ & & 0.005 & 0.015 & day $^{-1}$ \\
\hline Fungi & $p_{\text {lrate }}$ & Fungal litterfall rate & $(15)$ & 0.00225 & 0.00675 & day $^{-1}$ \\
\hline $\begin{array}{l}P \text { demand to } \\
\text { fungi }\end{array}$ & $p_{i, \text { rate }}$ & $\begin{array}{l}\text { Potential unit fungal } \\
\text { mycelia uptake rate } \\
\mathrm{PO}_{4}\end{array}$ & (19) & 0.00005 & 0.00015 & $\begin{array}{l}\mathrm{g} \mathrm{P} \mathrm{g} \mathrm{C}^{-1} \\
\mathrm{~m}^{-2} \text { day }^{-1}\end{array}$ \\
\hline $\begin{array}{l}N \text { demand to } \\
\text { fungi }\end{array}$ & $\begin{array}{l}n_{N H 4 \text { rate }} / \\
n_{N O 3 r a t e}\end{array}$ & $\begin{array}{l}\text { Potential unit fungal } \\
\text { mycelia uptake rate } \\
\mathrm{NH}_{4} / \mathrm{NO}_{3}\end{array}$ & & 0.0002 & 0.0006 & $\begin{array}{l}\mathrm{g} \mathrm{N} \mathrm{g} \mathrm{C}^{-1} \\
\mathrm{~m}^{-2} \text { day }^{-} \\
1 \mathrm{U}\end{array}$ \\
\hline
\end{tabular}




\begin{tabular}{|c|c|c|c|c|c|c|}
\hline $\begin{array}{l}N \text { demand to } \\
\text { fungi }\end{array}$ & $\begin{array}{l}n_{\text {olit,rate }} / \\
n_{\text {ohum,rate }}\end{array}$ & $\begin{array}{l}\text { Potential unit fungal } \\
\text { mycelia uptake rate } \\
\text { organic N, }\end{array}$ & & 0.00001 & 0.00003 & $\begin{array}{l}\mathrm{g} \mathrm{N} \mathrm{g} \mathrm{C}^{-1} \\
\mathrm{~m}^{-2} \mathrm{day}^{-1}\end{array}$ \\
\hline $\begin{array}{l}P \text { demand to } \\
\text { fungi }\end{array}$ & $p_{\text {cpfungimax }}$ & $\begin{array}{l}\text { Fungal maximum } \\
\mathrm{C} / \mathrm{P}\end{array}$ & (17) & 100 & 300 & $\mathrm{gC} \mathrm{gP}^{-1}$ \\
\hline P uptake & $p_{\text {iavail }}$ & $\begin{array}{l}\text { Maximum } \mathrm{PO}_{4} \\
\text { uptake fraction for } \\
\text { roots }\end{array}$ & $(21)$ & 0.004 & 0.012 & - \\
\hline $\begin{array}{l}P \text { demand to } \\
\text { fungi }\end{array}$ & $p_{\text {cpfungimin }}$ & $\begin{array}{l}\text { Fungal minimum } \\
\mathrm{C} / \mathrm{P}\end{array}$ & (22) & 50 & 150 & - \\
\hline $\begin{array}{l}P \text { demand to } \\
\text { fungi }\end{array}$ & $\begin{array}{l}p_{\text {Litterf }} / \\
p_{\text {Humusf }}\end{array}$ & $\begin{array}{l}\text { Potential unit fungal } \\
\text { mycelia uptake rate } \\
\text { organic } \mathrm{P}\end{array}$ & (23) & 0.00001 & 0.00003 & $\begin{array}{l}\mathrm{g} \mathrm{P} \mathrm{g} \mathrm{C}^{-1} \\
\mathrm{~m}^{-2} \text { day }^{-1}\end{array}$ \\
\hline $\begin{array}{l}P \\
\text { transformation }\end{array}$ & $c p_{m}$ & $\begin{array}{l}\mathrm{C} / \mathrm{P} \text { of non } \\
\text { symbiotic microbes }\end{array}$ & (A.3) & 175 & 525 & - \\
\hline $\begin{array}{l}\text { P demand to } \\
\text { plant }\end{array}$ & $c p_{\text {leaf, } \min }$ & Minimum C/P (leaf) & (A.9) & 110 & 330 & - \\
\hline $\begin{array}{l}P \text { demand to } \\
\text { plant }\end{array}$ & $\begin{array}{l}c p_{\text {stem, } \min } \\
/ c p_{\text {croot }} \\
\text { min }\end{array}$ & $\begin{array}{l}\text { Minimum } \mathrm{C} / \mathrm{P} \text {, for } \\
\text { stem and coarse } \\
\text { roots }\end{array}$ & (A.9) & 2000 & 6000 & - \\
\hline $\begin{array}{l}\text { P demand to } \\
\text { plant }\end{array}$ & $c p_{\text {root } \min }$ & $\begin{array}{l}\text { Minimum C/P ratio } \\
\text { (fine roots) }\end{array}$ & (A.9) & 200 & 600 & - \\
\hline Erosion & $p_{\text {base }}$ & $\begin{array}{l}\text { P concentration } \\
\text { scaling coefficient } \\
\text { for surface erosion } 1\end{array}$ & (A.14) & $1.35 \times 10^{-6}$ & $4.05 \times 10^{-4}$ & $\mathrm{mg} \mathrm{l}^{-1}$ \\
\hline Erosion & $p_{\Delta}$ & $\begin{array}{l}\text { P concentration } \\
\text { scaling coefficient } \\
\text { for surface erosion } 2\end{array}$ & (A.14) & $3.5 \times 10^{-6}$ & $1.05 \times 10^{-3}$ & $\mathrm{mg} \mathrm{l}^{-1}$ \\
\hline Erosion & $q_{t h r}$ & $\begin{array}{l}\text { Critical surface flow } \\
\text { rate for erosion }\end{array}$ & (A.14) & 0.5 & 15 & $\underset{1}{\mathrm{~mm}}$ day $^{-}$ \\
\hline $\begin{array}{l}P \\
\text { transformation }\end{array}$ & $m_{\text {retain }}$ & $\begin{array}{l}\text { Mobile coefficient } \\
\text { describing the } \\
\text { fraction of } \mathrm{P} \text { and } \mathrm{N} \\
\text { retained in an } \\
\text { internal mobile pool } \\
\text { when the plants goes } \\
\text { into dormancy }\end{array}$ & - & 0.1 & 0.3 & - \\
\hline
\end{tabular}


Table S.2. List of parameters with the largest influences on the simulated C, N and P fluxes and pools for the northernmost $64^{0} \mathrm{~N}$ region. Parameter importance was ranked by the given correlation coefficient (r) between the parameter and the model performance indicator.

\begin{tabular}{|c|c|c|c|c|c|c|c|c|}
\hline \multirow{3}{*}{$\begin{array}{l}\text { Variables } \\
\begin{array}{l}\text { Ecosystem C } \\
\text { change }\end{array}\end{array}$} & \multicolumn{4}{|c|}{$\begin{array}{l}\text { Parameters controlling dynamics } \\
\left(\mathrm{R}^{2}\right)\end{array}$} & \multicolumn{4}{|c|}{$\begin{array}{l}\text { Parameters controlling magnitude } \\
\text { (ME) }\end{array}$} \\
\hline & $c p_{m}$ & $I n i_{P, h}$ & $O_{\text {uptNhumus }}$ & & $O_{\text {uptNhumus }}$ & $c p_{m}$ & & \\
\hline & 0.23 & 0.2 & 0.2 & & 0.8 & 0.32 & & \\
\hline \multirow{2}{*}{$\begin{array}{l}\text { Total C } \\
\text { harvest }\end{array}$} & $O_{\text {uptNhumus }}$ & $c p_{m}$ & $p H_{o p t}$ & $p_{\text {fopt }}$ & $O_{\text {uptNhumus }}$ & $n_{c n, t h}$ & $p_{\text {fopt }}$ & \\
\hline & 0.29 & 0.27 & 0.2 & -0.2 & 0.83 & 0.25 & -0.2 & \\
\hline \multirow{2}{*}{$\begin{array}{l}\text { Plant C } \\
\text { change }\end{array}$} & $c p_{m}$ & $O_{\text {uptNhumus }}$ & & & $O_{\text {uptNhumus }}$ & $c p_{m}$ & $n_{c n, t h}$ & $p_{\text {fopt }}$ \\
\hline & 0.21 & 0.2 & & & 0.82 & 0.23 & 0.24 & -0.21 \\
\hline \multirow{2}{*}{$\begin{array}{l}\text { Soil C } \\
\text { change }\end{array}$} & $o_{\text {uptNhumus }}$ & $c p_{m}$ & & & $o_{\text {uptNhumus }}$ & $c p_{m}$ & $I n i_{P, h}$ & \\
\hline & 0.78 & 0.34 & & & 0.7 & 0.42 & 0.23 & \\
\hline \multirow{2}{*}{$\begin{array}{l}\text { Plant N } \\
\text { change }\end{array}$} & OuptNhumus $_{\text {up }}$ & $c p_{\text {leaf }, \min }$ & & & $O_{\text {uptNhumus }}$ & $n_{c n, t h}$ & $I n i_{P, h}$ & \\
\hline & 0.28 & 0.2 & & & 0.8 & -0.34 & -0.22 & \\
\hline \multirow{2}{*}{$\begin{array}{l}\text { Soil N } \\
\text { change }\end{array}$} & $O_{\text {uptNhumus }}$ & $c p_{m}$ & & & $O_{\text {uptNhumus }}$ & $I n i_{P, h}$ & $n_{c n, t h}$ & \\
\hline & 0.25 & 0.2 & & & -0.74 & 0.25 & 0.36 & \\
\hline \multirow{2}{*}{$\begin{array}{l}\text { Plant } P \\
\text { change }\end{array}$} & $O_{\text {uptNhumus }}$ & & & & $I n i_{P, h}$ & $c p_{m}$ & $p_{c p, t h}$ & \\
\hline & 0.31 & & & & 0.71 & 0.39 & -0.2 & \\
\hline \multirow{2}{*}{$\begin{array}{l}\text { Soil P } \\
\text { change }\end{array}$} & $O_{\text {uptNhumus }}$ & & & & $I n i_{P, h}$ & $c p_{m}$ & $p_{c p, o p t}$ & $p_{c p, t h}$ \\
\hline & 0.35 & & & & -0.70 & -0.4 & -0.41 & 0.2 \\
\hline \multirow{2}{*}{$\begin{array}{l}\text { Response of } \\
\mathrm{N} \text { on GPP }\end{array}$} & $I n i_{P, h}$ & $c p_{m}$ & $n_{c n, o p t}$ & & $I n i_{P, h}$ & $c p_{m}$ & $O_{\text {uptNhumus }}$ & \\
\hline & 0.38 & 0.29 & -0.26 & & -0.4 & -0.31 & 0.25 & \\
\hline \multirow{2}{*}{$\begin{array}{l}\text { Response of } \\
\mathrm{P} \text { on GPP }\end{array}$} & $I n i_{P, h}$ & $O_{\text {uptNhumus }}$ & $n_{c n, t h}$ & $p_{c p, o p t}$ & $I n i_{P, h}$ & $p_{c p, o p t}$ & $c p_{m}$ & $o_{\text {uptNhumus }}$ \\
\hline & -0.53 & 0.33 & 0.22 & -0.2 & 0.55 & 0.37 & 0.31 & -0.31 \\
\hline
\end{tabular}


Table S.3. List of parameters with the largest influences on the simulated C, N and P fluxes and pools for the southernmost $56^{\circ} \mathrm{N}$ region. Parameter importance was ranked by the given correlation coefficient (r) between the parameter and the model performance indicator.

\begin{tabular}{|c|c|c|c|c|c|c|c|c|}
\hline \multirow{3}{*}{\begin{tabular}{|l|} 
Variables \\
Ecosystem \\
C change \\
\end{tabular}} & \multicolumn{4}{|c|}{ Parameters controlling dynamics $\left(\mathrm{R}^{2}\right)$} & \multicolumn{4}{|c|}{$\begin{array}{l}\text { Parameters controlling magnitude } \\
(\mathrm{ME})\end{array}$} \\
\hline & & & & & $I n i_{P, h}$ & $O_{\text {uptNhumus }}$ & $c p_{m}$ & $O_{\text {uptPhumus }}$ \\
\hline & & & & & 0.45 & 0.28 & 0.25 & 0.24 \\
\hline \multirow{2}{*}{$\begin{array}{ll}\text { Total } & \mathrm{C} \\
\text { harvest } & \end{array}$} & $O_{\text {uptNhumus }}$ & $n_{c n, t h}$ & & & OuptNhumus & OuptPhumus $_{\text {un }}$ & $n_{c n, t h}$ & $p_{\text {fopt }}$ \\
\hline & 0.23 & 0.2 & & & 0.39 & 0.29 & 0.28 & -0.2 \\
\hline \multirow{2}{*}{$\begin{array}{ll}\begin{array}{l}\text { Plant } \\
\text { change }\end{array} & \mathrm{C} \\
\end{array}$} & $O_{\text {uptNhumus }}$ & & & & $O_{\text {uptNhumus }}$ & $O_{\text {uptPhumus }}$ & $n_{c n, t h}$ & $I n i_{P, h}$ \\
\hline & 0.35 & & & & 0.36 & 0.29 & 0.26 & 0.22 \\
\hline \multirow{2}{*}{$\begin{array}{ll}\begin{array}{l}\text { Soil } \\
\text { change }\end{array} & \mathrm{C}\end{array}$} & $I n i_{P, h}$ & $c p_{m}$ & & & $I n i_{P, h}$ & $c p_{m}$ & & \\
\hline & 0.39 & 0.25 & & & 0.61 & 0.39 & & \\
\hline \multirow{2}{*}{$\begin{array}{ll}\begin{array}{l}\text { Plant } \\
\text { change }\end{array} & \mathrm{N} \\
\end{array}$} & $I n i_{P, h}$ & $c p_{m}$ & & & $I n i_{P, h}$ & $O_{\text {uptNhumus }}$ & $c p_{m}$ & $p_{\text {fopt }}$ \\
\hline & 0.33 & 0.23 & & & -0.61 & 0.4 & -0.34 & -0.26 \\
\hline \multirow{2}{*}{$\begin{array}{ll}\begin{array}{l}\text { Soil } \\
\text { change }\end{array} & \mathrm{N}\end{array}$} & $I n i_{P, h}$ & $c p_{m}$ & & & $I n i_{P, h}$ & $c p_{m}$ & $O_{\text {uptNhumus }}$ & \\
\hline & 0.27 & 0.24 & & & 0.61 & 0.34 & -0.26 & \\
\hline \multirow{2}{*}{$\begin{array}{ll}\text { Plant } & P \\
\text { change }\end{array}$} & $I n i_{P, h}$ & $o_{\text {uptNhumus }}$ & $c p_{\text {min,leaf }}$ & $c p_{\text {min,root }}$ & $I n i_{P, h}$ & $c p_{m}$ & & \\
\hline & -0.37 & 0.27 & -0.27 & -0.22 & 0.78 & 0.41 & & \\
\hline \multirow{2}{*}{$\begin{array}{ll}\begin{array}{l}\text { Soil } \\
\text { change }\end{array} & \mathrm{P} \\
\end{array}$} & $I n i_{P, h}$ & $c p_{\text {min,root }}$ & $o_{\text {uptNhumus }}$ & $c p_{\text {min,leaf }}$ & $I n i_{P, h}$ & $c p_{m}$ & & \\
\hline & -0.4 & -0.28 & 0.27 & -0.27 & -0.77 & -0.4 & & \\
\hline \multirow{2}{*}{$\begin{array}{l}\text { Response } \\
\text { of N on } \\
\text { GPP }\end{array}$} & $n_{c n, o p t}$ & & & & $I n i_{P, h}$ & $c p_{m}$ & $O_{\text {uptNhumus }}$ & \\
\hline & -0.3 & & & & -0.68 & -0.24 & 0.22 & \\
\hline \multirow{2}{*}{$\begin{array}{l}\text { Response } \\
\text { of P on GPP }\end{array}$} & $I n i_{P, h}$ & $c p_{m}$ & $o_{\text {uptNhumus }}$ & & $I n i_{P, h}$ & $c p_{m}$ & $p_{c p, o p t}$ & \\
\hline & -0.75 & -0.25 & 0.21 & & 0.70 & 0.26 & 0.23 & \\
\hline
\end{tabular}


Table S.4. List of parameters with the largest influences on the simulated C, N and P fluxes and pools for the northernmost $64^{\circ} \mathrm{N}$ region, excluding initial soil $\mathrm{P}$ and short-cut uptake coefficients for $\mathrm{N}$ and $\mathrm{P}$. Parameter importance was ranked by the given correlation coefficient (r) between the parameter and the model performance indicator.

\begin{tabular}{|c|c|c|c|c|c|c|c|c|}
\hline \multirow{3}{*}{\begin{tabular}{|l|} 
Variables \\
Ecosystem \\
C change \\
\end{tabular}} & \multicolumn{4}{|c|}{$\begin{array}{l}\text { Parameters controlling dynamics } \\
\left(\mathrm{R}^{2}\right)\end{array}$} & \multicolumn{4}{|c|}{$\begin{array}{l}\text { Parameters controlling magnitude } \\
(\mathrm{ME})\end{array}$} \\
\hline & $n_{\text {NO3rate }}$ & $p_{\text {Humusf }}$ & $m_{\text {retain }}$ & $c p_{\text {min,root }}$ & $n_{c n, t h}$ & $k_{r m}$ & $p_{\text {fopt }}$ & $n_{\text {NHArate }}$ \\
\hline & 0.35 & -0.23 & 0.23 & -0.27 & 0.7 & 0.49 & -0.38 & -0.37 \\
\hline \multirow{2}{*}{$\begin{array}{ll}\text { Total } & \mathrm{C} \\
\text { harvest } & \end{array}$} & $p_{\text {fopt }}$ & $n_{c n, t h}$ & $c p_{\text {min,leaf }}$ & & $n_{c n, t h}$ & $k_{r m}$ & $p_{\text {fopt }}$ & $n_{\text {NHArate }}$ \\
\hline & -0.49 & 0.47 & -0.27 & & 0.63 & 0.57 & -0.4 & -0.35 \\
\hline \multirow{2}{*}{$\begin{array}{ll}\begin{array}{l}\text { Plant } \\
\text { change }\end{array} \\
\end{array}$} & $n_{c n, t h}$ & $p_{\text {fopt }}$ & & & $n_{c n, t h}$ & $k_{r m}$ & $p_{\text {fopt }}$ & $n_{\text {NHArate }}$ \\
\hline & 0.47 & -0.3 & & & 0.63 & 0.54 & -0.43 & -0.36 \\
\hline \multirow{2}{*}{$\begin{array}{ll}\begin{array}{l}\text { Soil } \\
\text { change }\end{array} & \mathrm{C} \\
\end{array}$} & $p_{\text {lrate }}$ & $c p_{\text {min, root }}$ & $n_{c n, o p t}$ & $p_{c p, o p t}$ & $n_{c n, t h}$ & $n_{\text {NH4rate }}$ & $c p_{\text {min,croot }}$ & $c_{50, a d s}$ \\
\hline & -0.29 & -0.28 & 0.26 & -0.22 & 0.72 & -0.31 & -0.29 & -0.22 \\
\hline \multirow{2}{*}{$\begin{array}{ll}\begin{array}{l}\text { Plant } \\
\text { change }\end{array} & \mathrm{N} \\
\end{array}$} & $n_{c n, t h}$ & $c_{50, a d s}$ & $p_{\text {iavail }}$ & $n_{\text {avail }}$ & $n_{c n, t h}$ & $p_{\text {lrate }}$ & $q_{t h r}$ & $k_{w}$ \\
\hline & -0.42 & 0.28 & 0.25 & 0.25 & -0.86 & -0.3 & 0.28 & -0.25 \\
\hline \multirow{2}{*}{$\begin{array}{ll}\begin{array}{l}\text { Soil } \\
\text { change }\end{array} & \mathrm{N} \\
\end{array}$} & $c p_{\text {min,stem }}$ & $p_{c p, o p t}$ & $p_{\text {iavail }}$ & $p_{\text {fopt }}$ & $n_{c n, t h}$ & $c_{50, a d s}$ & $p_{c p, t h}$ & $p_{\text {lrate }}$ \\
\hline & 0.23 & 0.23 & -0.22 & 0.2 & 0.78 & -0.26 & 0.22 & 0.23 \\
\hline \multirow{2}{*}{$\begin{array}{ll}\begin{array}{l}\text { Plant } \\
\text { change }\end{array} & \mathrm{P} \\
\end{array}$} & $c p_{\text {min, leaf }}$ & $c p_{m}$ & $n_{c n, o p t}$ & $p_{\max , a d s}$ & $c p_{m}$ & $p_{\max , a d s}$ & $p H_{o p t}$ & $k_{r m}$ \\
\hline & -0.71 & -0.54 & -0.36 & 0.23 & 0.84 & -0.32 & -0.3 & 0.28 \\
\hline \multirow{2}{*}{$\begin{array}{ll}\begin{array}{l}\text { Soil } \\
\text { change }\end{array} & \mathrm{P} \\
\end{array}$} & $c p_{\text {min, leaf }}$ & $c p_{m}$ & $n_{c n, o p t}$ & $p_{c p, o p t}$ & $c p_{m}$ & $k_{r m}$ & $p H_{o p t}$ & $p_{\max , a d s}$ \\
\hline & -0.73 & -0.56 & -0.32 & -0.25 & -0.83 & -0.32 & 0.32 & 0.30 \\
\hline \multirow{2}{*}{$\begin{array}{l}\text { Response } \\
\text { of } \mathrm{N} \text { on } \\
\text { GPP }\end{array}$} & $n_{c n, o p t}$ & $n_{c n, t h}$ & $p_{i, \text { rate }}$ & $p_{c p, t h}$ & $n_{c n, o p t}$ & $n_{c n, t h}$ & $p_{i, \text { rate }}$ & \\
\hline & -0.53 & 0.31 & -0.31 & -0.22 & 0.47 & -0.37 & 0.29 & \\
\hline \multirow{2}{*}{$\begin{array}{l}\text { Response } \\
\text { of } \mathrm{P} \text { on } \\
\mathrm{GPP}\end{array}$} & $p_{c p, o p t}$ & $c p_{\text {min,leaf }}$ & $c p_{m}$ & $p_{c p, t h}$ & $p_{c p, o p t}$ & $c p_{\text {min,leaf }}$ & $p_{\text {Humusf }}$ & \\
\hline & -0.64 & -0.39 & -0.38 & 0.26 & 0.53 & -0.5 & -0.38 & \\
\hline
\end{tabular}



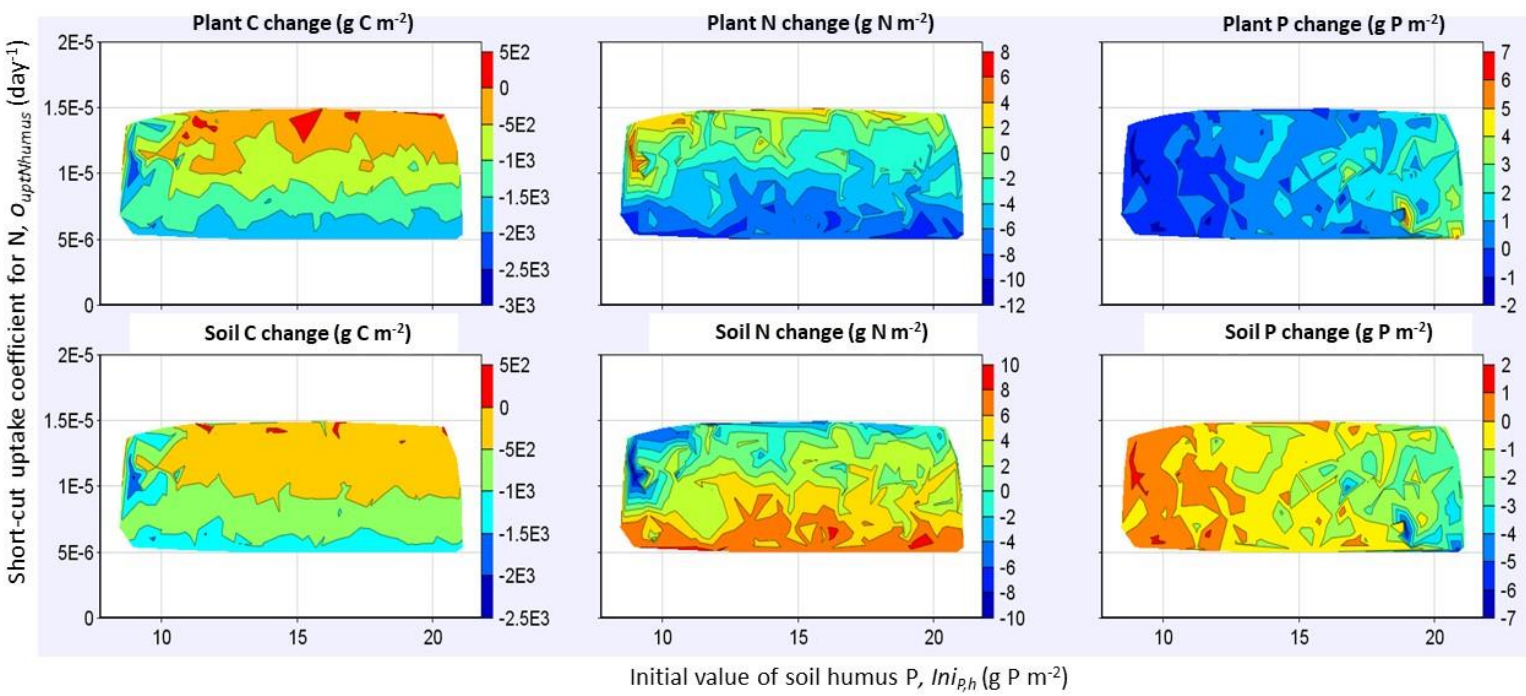

Fig. S.1 Plant and soil changes in C, N and P in relation to the two most influential parameters (initial soil humus $\mathrm{P}\left(\operatorname{Ini}_{P, h}\right)$ and the coefficient for short-cut $\mathrm{N}$ uptake from humus ( $\left.o_{\text {uptNhumus }}\right)$ ) for the northernmost region $\left(64^{0} \mathrm{~N}\right)$.

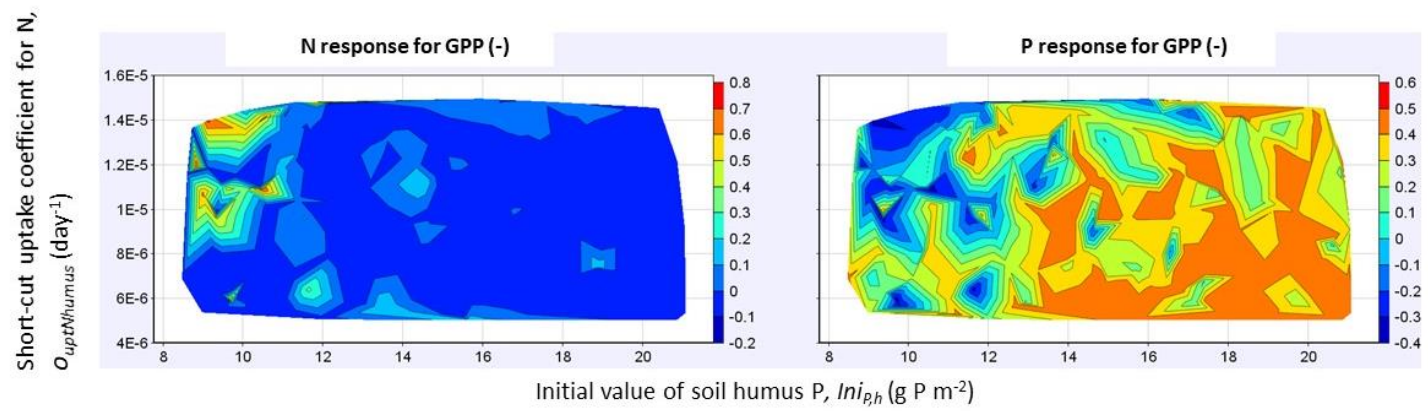

Fig. S.2 Responses of N and P on GPP in relation to the two most influential parameters (initial soil humus $\mathrm{P}\left(\operatorname{Ini}_{P, h}\right)$ and the coefficient for short-cut $\mathrm{N}$ uptake from humus $\left(o_{\text {uptNhumus }}\right)$ ) for the northernmost region $\left(64^{0} \mathrm{~N}\right)$. 


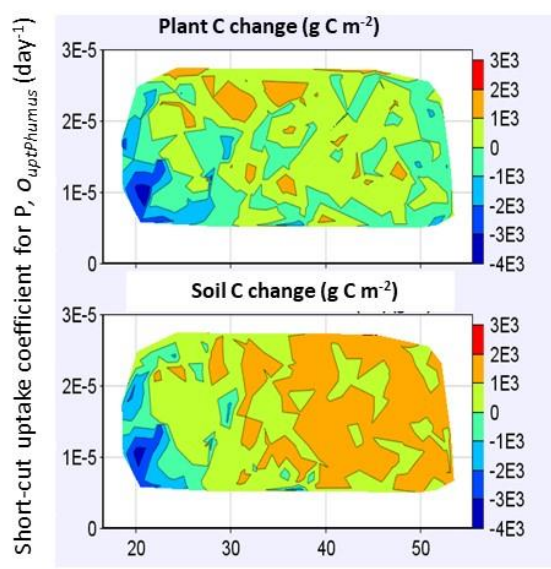

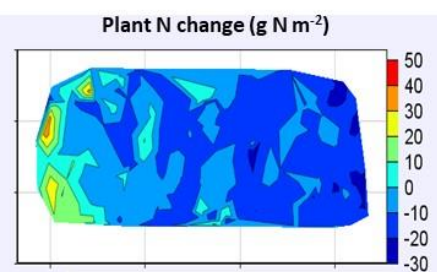

Soil $\mathrm{N}$ change $\left(\mathrm{g} \mathrm{N} \mathrm{m}^{-2}\right)$

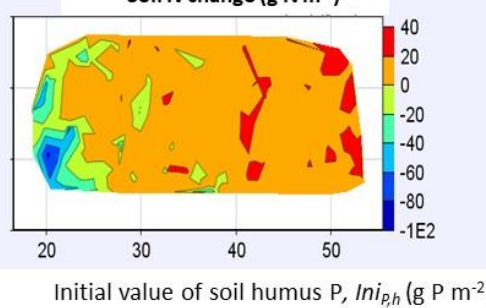

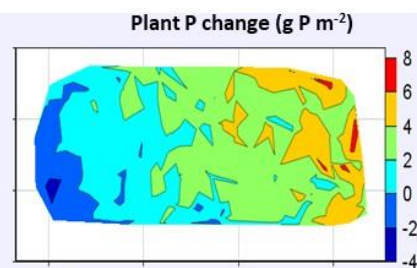

Soil P change ( $\left.\mathrm{g} \mathrm{P} \mathrm{m}^{-2}\right)$

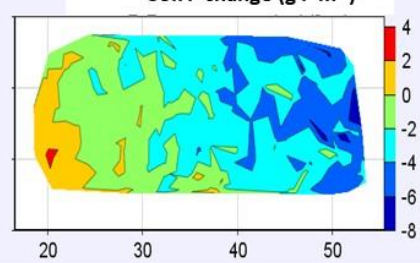

Fig. S.3 Plant and soil changes in $\mathrm{C}, \mathrm{N}$ and $\mathrm{P}$ in relation to the two most influential parameters (initial soil humus $\mathrm{P}\left(\operatorname{Ini}_{P, h}\right)$ and the coefficient for short-cut $\mathrm{P}$ uptake from humus $\left(o_{\text {uptPhumus }}\right)$ ) for the southernmost region $\left(56^{0} \mathrm{~N}\right)$.

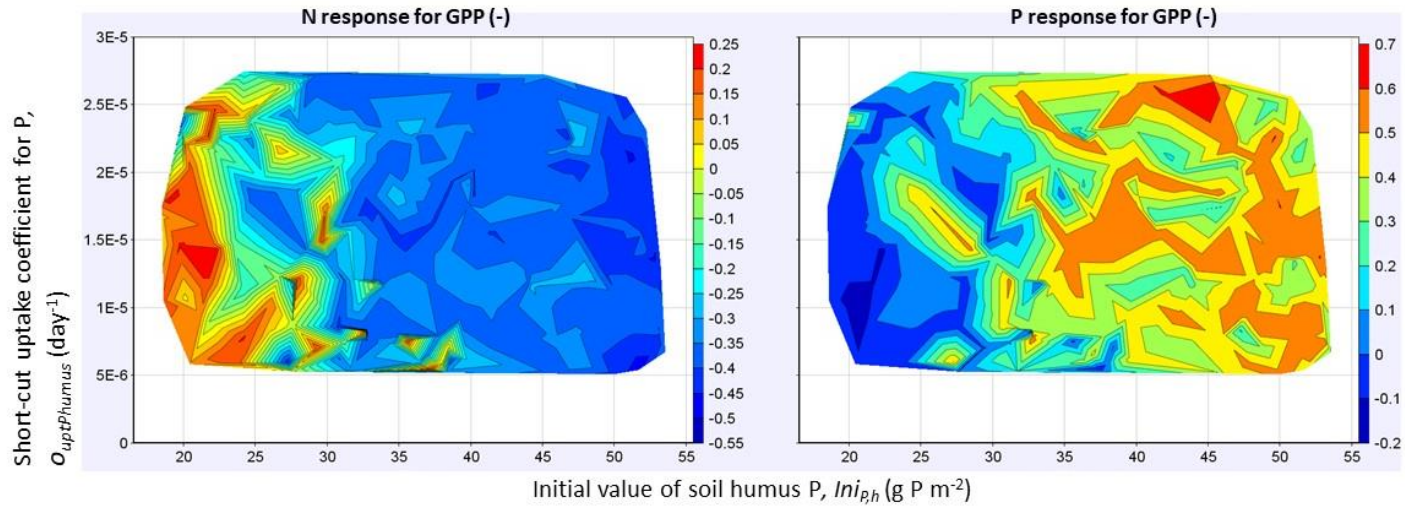

Fig. S.4 Responses of N and P on for GPP in relation to the two most influential parameters (initial soil humus $\mathrm{P}\left(\operatorname{Ini}_{P, h}\right)$ and the coefficient for short-cut $\mathrm{P}$ uptake from humus $\left(o_{\text {uptPhumus }}\right)$ ) for the southernmost region $\left(56^{0} \mathrm{~N}\right)$.

\section{Data availability}

The files used to generate the sensitivity analysis were archived at Zenodo (https://doi.org/10.5281/zenodo.4291963 ), also they will be made available from the CoupModel webpage www.coupmodel.com. 